\title{
Difficult intubation in a patient with vallecular cyst
}

\author{
Harikrishnan Kothandan ${ }^{1}$, MBBS, FAnzCA, Vui Kian $\underline{\mathrm{Ho}^{1}}$, MBBS, Mmed, Yew Meng $\underline{\mathrm{Chan}}{ }^{2}$, MBBS, FRCs, \\ Theodore Wong ${ }^{1}$, MD, FRCPC
}

\begin{abstract}
Although rare, vallecular cysts can have catastrophic consequences in an anaesthetised patient if airway management is inappropriate. We report a case of difficult intubation in a 46-year-old man with a vallecular cyst, and detail the methods and strategies for successful endotracheal tube insertion. Following a review of the current literature, we also discuss airway management options in adult patients with vallecular cysts.
\end{abstract}

Keywords: airway obstruction, anaesthesia, difficult airway, fibreoptic bronchoscope, vallecular cyst

\section{INTRODUCTION}

Vallecular cysts can cause extreme problems in securing the airway. Although rare, cases of unanticipated difficult intubation from asymptomatic vallecular cysts have been well documented in the literature. ${ }^{(1-4)}$ Meticulous airway assessment and planning is mandatory in the anaesthetisation of patients with vallecular cysts. We describe a case of difficult intubation in a patient with a vallecular cyst and discuss the airway management options following a review of the current literature.

\section{CASE REPORT}

The hospital's ethics committee granted approval for the publication of this case report and waiver of informed consent (CIRB Ref No: 2011/417/D). We report the anaesthetic management of a 46-year-old male ex-smoker who consumed 30 packets of cigarettes annually, until he quit smoking at the age of 44 . The patient, who presented to us for excision of a vallecular cyst, was obese with a body mass index of $32.3 \mathrm{~kg} / \mathrm{m}^{2}$, and had unremarkable baseline investigations and no significant comorbidities.

The patient had first presented to a general practitioner with complaints of a sensation of lumps in both nostrils, with occasional nasal sensitivity and sneezing, over the period of a few months. A working diagnosis of allergic rhinitis was made then, but the course of nasal steroids prescribed failed to alleviate his symptoms. He was subsequently referred to the otolaryngological outpatient clinic and diagnosed with deviated nasal septum and inferior turbinate hypertrophy. A vallecular cyst measuring $2.5 \mathrm{~cm}$ in diameter (Fig. 1) was incidentally found on nasendoscopy. The patient consented to excision biopsy of the vallecular cyst.

Pre-anaesthetic assessment done one week prior to the surgery revealed no symptoms of airway obstruction or respiratory difficulties. Airway assessment indicated a Mallampati score of 4, with normal neck movements and thyromental distance. The patient's interincisor distance was $5 \mathrm{~cm}$, and a few molar teeth were missing. Physical examination was normal. He was counselled regarding the possibility of a difficult intubation, which could necessitate an awake fibreoptic intubation.
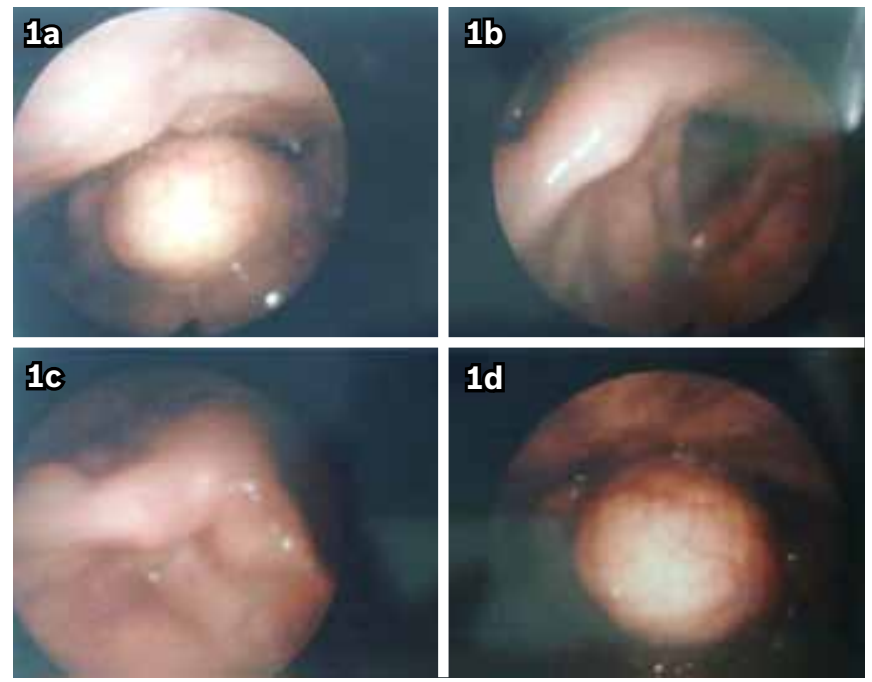

Fig. 1 Nasendoscopic images show (a \& d) a vallecular cyst measuring $2.5 \mathrm{~cm}$ in diameter obstructing the view of the vocal cords; and (b \& c) the vocal cords after successful negotiation past the vallecular cyst.

On the day of surgery, the patient was reassessed and particular attention was paid to his airway. His case notes and nasendoscopic images were also reviewed. Based on the assessment and discussion with the otolaryngologist, it was decided that the asleep intubation technique using the Macintosh laryngoscope while maintaining spontaneous respiration would be attempted first. The GlideScope video laryngoscope (Verathon Inc, Bothell, WA, USA) and difficult intubation trolley, which included McCoy blades, were prepared. If this failed, the alternative was to wake the patient and proceed with an awake fibreoptic bronchoscope. A rigid laryngoscopy set was made immediately available in the event of an airway crisis, and the surgeons were in sterile gloves and gown by the time of induction. Large-bore rigid suction catheters were also made immediately available in case of cyst rupture and bleeding.

The patient was placed in a supine position on a jelly doughnut. Standard monitors were applied and the patient was preoxygenated. Induction of anaesthesia was achieved with

${ }^{1}$ Department of Anaesthesiology, ${ }^{2}$ Department of ENT Surgery, Singapore General Hospital, Singapore

Correspondence: Dr Harikrishnan Kothandan, Consultant, Department of Anaesthesiology, Singapore General Hospital, Outram Road, Singapore 169608. sudhari71@gmail.com 
remifentanil (GlaxoSmithKline, SPA Pharma, Verona, Italy) infusion at $0.1 \mathrm{mcg} / \mathrm{kg} / \mathrm{min}$ and intravenous propofol (Fresenius $\mathrm{Kab} \mathrm{Ag}$, Bad Homburg, Germany) at $50 \mathrm{mg}$. Spontaneous ventilation was maintained and administration of desflurane via face mask was started. An end-tidal desflurane concentration of around $6 \%$ was achieved and loss of eyelash reflex was confirmed before direct laryngoscopy was attempted with a Macintosh 3 blade. An anaesthesia registrar with more than four years of experience performed the first attempt. An anaesthesia consultant with more than ten years of experience and more than 100 GlideScope-assisted endotracheal intubations performed all the subsequent attempts. However, despite the attempts of the two anaesthetists, who both optimised the patient's position with neck flexion, head extension and external laryngeal pressure, the vocal cords could not be visualised due to obstruction by the fleshy, nonpedunculated vallecular cyst.

The patient's ventilation was assisted by a bag and mask with a size 4 oral airway. There was some initial difficulty with mask ventilation, with only small tidal volumes achieved despite optimal positioning. Tidal volumes subsequently improved when the patient resumed regular, spontaneous breathing. Anaesthesia was maintained with remifentanil infusion and administration of desflurane via face mask. Further attempts with a GlideScope video laryngoscope (size 3) and a McCoy blade (size 4) yielded a Cormack-Lehane grade IV view. The cyst could not be moved out of the way by the laryngoscope or the microlaryngeal tube. A blind intubation attempt with gum elastic bougie-assisted insertion of a size $5.0 \mathrm{~mm}$ (internal diameter) Rush $^{\circledR}$ microlaryngeal tube (Rush Inc, Duluth, GA, USA) resulted in oesophageal intubations. Further attempts at asleep intubation were aborted and the patient was awoken.

The patient was then prepared for awake fibreoptic intubation. A co-phenylcaine forte spray (ENT Technologies, Hawthorn East, VIC, Australia; lignocaine hydrochloride 5\%, phenylephrine hydrochloride $0.5 \%$ ) was applied to the nostrils. $5 \mathrm{~mL}$ of nebulised lignocaine 2\% (Pfizer, Bentley, WA, Australia) was administered and a transtracheal block was performed using $3 \mathrm{~mL}$ of lignocaine $2 \%$. Awake fibreoptic intubation proceeded uneventfully via the right nostril, with the fibreoptic scope successfully negotiating past the vallecular cyst for clear visualisation of the patient's vocal cords. His trachea was intubated using a reinforced tracheal tube with an internal diameter of $6.0 \mathrm{~mm}$. After confirmation of successful tracheal tube placement, anaesthesia and paralysis were induced.

The otolaryngologist found a bilobed vallecular cyst that extended to the epiglottic base and excised it uneventfully. Dexamethasone $12 \mathrm{mg}$ (Wasserburger Arzneimittelwerk GmbH, Wasserburg, Germany) was given intravenously to limit airway oedema. The patient had an uneventful awake extubation at the end of the operation and was transferred to the high dependency unit with intranasal supplemental oxygen at $2 \mathrm{~L} / \mathrm{min}$. Standard monitoring, including pulse oximetry, was applied throughout the period of observation in the high dependency unit.

\section{DISCUSSION}

Vallecular cysts are formed when the duct of a mucous gland or lingual tonsillar crypt becomes dilated due to obstruction from inflammation, irritation or trauma. ${ }^{(5-7)}$ DeSanto et al classically subdivided laryngeal cysts into ductal, saccular and thyroid cartilage foraminal cysts, ${ }^{(5,7,8)}$ while more recent classification systems have described congenital, retention, inclusion and lymphoepithelial cysts. ${ }^{(7,9)}$ The most common form of laryngeal cysts is the ductal cyst, which frequently presents at the true vocal fold, followed by the epiglottis and vallecula. ${ }^{(6)}$

Clinically, there is a bimodal distribution in the age of presentation, thus suggesting two forms of vallecular cysts - adult and paediatric..$^{(6,10)}$ While vallecular cysts in children are rare, there is a higher prevalence in neonates and infants. ${ }^{(5,11)}$ There are also reports of cysts detected during the antenatal period via sonography and magnetic resonance imaging. ${ }^{(5,10,12)}$ Symptoms in children include stridor, chest wall retraction, dyspnoea, abnormal cry, poor feeding, failure to thrive and apnoea. ${ }^{(10)}$ These patients may be asymptomatic at birth, but later develop obstructive symptoms that are occasionally exacerbated by an upper respiratory tract infection. ${ }^{(10)}$ Airway obstruction in neonates and infants poses a particular challenge for the anaesthetist, as children have less physiological reserves and much smaller airways. Moreover, laryngomalacia may add to the patient's symptoms as well as the complexity involved in securing the airway. ${ }^{(13,14)}$ If a vallecular cyst is detected antenatally, elective delivery should be performed in a tertiary referral centre, where a multidisciplinary team can be present and the requisite equipment are available to secure the airway emergently and remove the cyst. ${ }^{(5,12,15)}$

Vallecular cysts in adults are even rarer, with the peak incidence in the fifth decade and occurring more frequently in men. ${ }^{(2,6,8,9)}$ While these patients may present with globus, voice changes, dysphagia, odynophagia, stridor and dyspnoea, the majority are asymptomatic and incidentally diagnosed on routine laryngeal examination. ${ }^{(6,9)}$ Symptoms may manifest after the vallecular cyst has become infected and an abscess has formed. ${ }^{(3,4)}$ The incidence of vallecular cysts on laryngoscopy is estimated to be between 1 in 1250 to 1 in 4200.(6) Asymptomatic vallecular cysts are a known cause of unanticipated difficult intubation and are well documented in case reports. ${ }^{(1-3)}$ Moreover, vallecular cysts can pose a ventilatory challenge, especially with pedunculated cysts that can result in a 'ball-valve' effect. ${ }^{(2)}$ Surgical management of a symptomatic vallecular cyst is complete removal by marsupialisation, deroofing or excision with $\mathrm{CO}_{2}$ laser or electrocautery. ${ }^{(6,16)}$ Aspiration of the cyst is likely to result in recurrence and thus not recommended as a definitive treatment. ${ }^{(16)}$

We summarise three recent case reports on vallecular cysts causing difficult intubation to illustrate the possible solutions and pitfalls in airway management in such patients. Kamble et $\mathrm{al}^{(2)}$ described a 47-year-old obese female smoker who presented for elective right knee arthroscopy. She had hypertension, 
gastro-oesophageal reflux and an uneventful general anaesthetic via laryngeal mask a year before. She was edentulous with no airway or respiratory signs or symptoms. Rapid sequence induction with cricoid pressure was instituted. A right-sided pedunculated vallecular cyst measuring $2 \mathrm{~cm}$, which completely obstructed any view of the larynx or epiglottis, was found on direct laryngoscopy with a Macintosh 3 blade. Intubation was unsuccessful despite multiple attempts by two anaesthesiologists using Macintosh 3 and Miller 2 blades, and optimal positioning and laryngeal pressure. Ventilation of the patient was difficult, even with an oropharyngeal airway, and was complicated by desaturation and laryngospasm. A second dose of succinylcholine was administered and intubation was reattempted with a Miller 2 blade and a styletted endotracheal tube. The tube was used to push the cyst aside, revealing a limited view of the laryngeal inlet, and the trachea was intubated. The vallecular cyst was removed intraoperatively and dexamethasone was administered following an urgent otolaryngology consult. The patient's subsequent extubation was uncomplicated.

Rivo and Matot $^{(1)}$ reported the case of a 24-year-old man who presented for an emergency appendectomy. He had undergone uneventful general anaesthesia for posterior spine fusion ten years prior and was otherwise healthy. There was no airway or respiratory signs or symptoms found during preoperative assessment. Rapid sequence induction with cricoid pressure was performed after preoxygenation, and direct laryngoscopy with a Macintosh 3 blade revealed a left-sided vallecular cyst measuring $5 \mathrm{~cm}$, which completely obscured the glottis. Four attempts at intubation with a styletted endotracheal tube, optimal sniff position and laryngeal pressure, and Miller size 3 and 4 blades were unsuccessful. Mask ventilation was adequate and the patient was woken up when he started to breathe spontaneously. Transnasal awake fibreoptic intubation was performed successfully at the first attempt. The vallecular cyst was removed intraoperatively by the otolaryngologist and dexamethasone was administered. At the end of the operation, the patient was extubated after a successful leak test. However, he developed stridor postoperatively, which resolved with dexamethasone and nebulised adrenaline.

Walshe et $\mathrm{al}^{(3)}$ described a 31-year-old male smoker and intravenous drug user who presented with complaints of dysphagia, anorexia and weight loss of one-year duration, as well as three weeks of dysphonia and dyspnoea on exertion. Physical examination of his airway was unremarkable. However, flexible fibreoptic nasolaryngoscopy revealed a vallecular mass. Inhalational induction with sevoflurane in $100 \%$ oxygen was performed and boluses of propofol were added to ensure adequate depth of anaesthesia while maintaining spontaneous respiration. Three attempts at intubation with Macintosh 3, McCoy 3 and Miller blades were unsuccessful. The patient remained easy to mask ventilate. Finally, the trachea was intubated with a rigid laryngoscope by the otolaryngologist, who used the scope to displace the cyst in order to visualise the glottis. Pus was aspirated from the cyst and dexamethasone was given. The patient was extubated uneventfully.

As was the case in Walshe et al's report, ${ }^{(3)}$ we were aware of the potentially difficult airway in our patient and thus employed intubation techniques that maintained spontaneous respiration in order to safely secure the airway. Possible options, whether asleep or awake, include direct laryngoscopy, video laryngoscopy, rigid laryngoscopy (by an otolaryngologist) and flexible scopes. If the cyst obscures the laryngeal view, these approaches may be successful if the cyst can be displaced to one side using either the scope or the tube. ${ }^{(1-3)}$ The rigid laryngoscope, for example, is longer than the anaesthetic laryngoscope, and is thus able to pass beyond and displace the cyst to visualise the vocal cords. ${ }^{(3)}$ Scopes with lens at the tip, such as the Bonfils intubation fibrescope and flexible fibreoptic bronchoscope, have an advantage, since it is easier to manoeuvre the instrument past the obstacle instead of forcefully displacing it. ${ }^{(1)}$ Other documented procedures that may aid in vocal cord visualisation include aspiration of cystic contents with a spinal needle to decrease the size of the cyst, and retrograde tracheal intubation. ${ }^{(1)}$ While blind intubation may occasionally be successful, it is imperative to avoid multiple attempts, as this may cause airway oedema, potentially exacerbating any airway obstruction. Moreover, there has been at least one case report where repeated blind attempts resulted in airway bleeding sufficient for the operation to be abandoned. ${ }^{(1)}$ In addition, blind attempts might rupture the cyst and cause airway compromise. In cases where a large vallecular cyst has been documented preoperatively, it is prudent to review computed tomography or magnetic resonance images of the patient's neck in order to evaluate the extent of the cyst, including the presence of any perilesional oedema and anatomical distortion. ${ }^{(4)}$ If radiological images were available in our patient, they would have provided invaluable information in the planning of airway management.

In conclusion, vallecular cysts can cause extreme problems in securing the airway. Meticulous airway assessment and contingency planning are mandatory, as with any difficult airway scenario. Close attention to logistics and the immediate availability of an otolaryngologist is vital. The use of Bonfils intubation fibrescope or a flexible fibreoptic bronchoscope in a spontaneously breathing patient who is either asleep or awake might increase the success rate compared to the use of direct or other video laryngoscopy techniques.

\section{REFERENCES}

1. Rivo J, Matot I. Asymptomatic vallecular cyst: airway management considerations. J Clin Anesth 2001; 13:383-6.

2. Kamble VA, Lilly RB, Gross JB. Unanticipated difficult intubation as a result of an asymptomatic vallecular cyst. Anesthesiology 1999; 91:872-3.

3. Walshe CM, Jonas $\mathrm{N}$, Rohan D. Vallecular cyst causing a difficult intubation. Br J Anaesth 2009; 102:565.

4. Berger G, Averbuch E, Zilka K, Berger R, Ophir D. Adult vallecular cyst: thirteen-year experience. Otolaryngol Head Neck Surg 2008; 138:321-7.

5. Cuillier F, Samperiz S, Testud R, Fossati P. Antenatal diagnosis and management of a vallecular cyst. Ultrasound Obstet Gynecol 2002; 20:623-6.

6. Romak J, Olsen SM, Koch CA, Ekbom DC. Bilateral vallecular cysts as a 
cause of Dysphagia: case report and literature review. Int J Otolaryngol 2010; 2010:697583. Epub 2010 Dec 12.

7. Forte $\mathrm{V}$, Fuoco $\mathrm{G}$, James A. A new classification system for congenital laryngeal cysts. Laryngoscope 2004; 114:1123-7.

8. DeSanto LW, Devine KD, Weiland LH. Cysts of the larynx--classification. Laryngoscope 1970; 80:145-76.

9. Arens C, Glanz H, Kleinsasser O. Clinical and morphological aspects of laryngeal cysts. Eur Arch Otorhinolaryngol 1997; 254:430-6.

10. Mahajan V, Mathew JL, Singh M, Gupta R, Das A. Vallecular cyst-revisited. Indian J Pediatr 2008; 75:1081-2.

11. Liu HC, Lee KS, Hsu CH, Hung HY. Neonatal vallecular cyst: report of eleven cases. Changgeng Yi Xue Za Zhi 1999; 22:615-20.

12. Cuillier F, Testud R, Samperiz S, Fossati P. [Prenatal diagnosis at 25 weeks gestation and neonatal management of a vallecular cyst]. Ann Otolaryngol Chir Cervicofac 2002; 119:293-5. French.

13. Cheng KS, Ng JM, Li HY, Hartigan PM. Vallecular cyst and laryngomalacia in infants: report of six cases and airway management. Anesth Analg 2002; 95:1248-50.

14. Hsieh WS, Yang PH, Wong KS, Li HY, Wang EC, Yeh TF. Vallecular cyst: an uncommon cause of stridor in newborn infants. Eur J Pediatr 2000; 159:79-81.

15. Busuttil M, Einaudi MA, Hassid S, et al. Congenital laryngeal cyst: benefits of prenatal diagnosis and multidisciplinary perinatal management. Fetal Diagn Ther 2004; 19:373-6.

16. Bhandary S. Innovative Surgical Technique in the Management of Vallecular Cyst. Online J Health Allied Scs 2003; 2:2. 